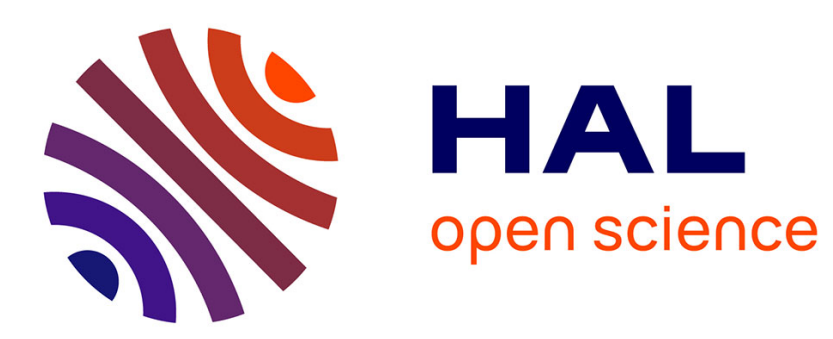

\title{
Empirical versus time stepping with embedded error control for density-driven flow in porous media
}

\author{
A. Younes, P. Ackerer
}

\section{To cite this version:}

A. Younes, P. Ackerer. Empirical versus time stepping with embedded error control for density-driven flow in porous media. Water Resources Research, 2010, 46, pp.W08523. 10.1029/2009WR008229 . insu-00579427

\section{HAL Id: insu-00579427 https://hal-insu.archives-ouvertes.fr/insu-00579427}

Submitted on 12 Mar 2021

HAL is a multi-disciplinary open access archive for the deposit and dissemination of scientific research documents, whether they are published or not. The documents may come from teaching and research institutions in France or abroad, or from public or private research centers.
L'archive ouverte pluridisciplinaire HAL, est destinée au dépôt et à la diffusion de documents scientifiques de niveau recherche, publiés ou non, émanant des établissements d'enseignement et de recherche français ou étrangers, des laboratoires publics ou privés. 


\title{
Empirical versus time stepping with embedded error control for density-driven flow in porous media
}

\author{
Anis Younes ${ }^{1}$ and Philippe Ackerer ${ }^{1}$ \\ Received 21 May 2009; revised 18 March 2010; accepted 2 April 2010; published 11 August 2010.
}

[1] Modeling density-driven flow in porous media may require very long computational time due to the nonlinear coupling between flow and transport equations. Time stepping schemes are often used to adapt the time step size in order to reduce the computational cost of the simulation. In this work, the empirical time stepping scheme which adapts the time step size according to the performance of the iterative nonlinear solver is compared to an adaptive time stepping scheme where the time step length is controlled by the temporal truncation error. Results of the simulations of the Elder problem show that (1) the empirical time stepping scheme can lead to inaccurate results even with a small convergence criterion, (2) accurate results are obtained when the time step size selection is based on the truncation error control, (3) a non iterative scheme with proper time step management can be faster and leads to more accurate solution than the standard iterative procedure with the empirical time stepping and (4) the temporal truncation error can have a significant effect on the results and can be considered as one of the reasons for the differences observed in the Elder numerical results.

Citation: Younes, A., and P. Ackerer (2010), Empirical versus time stepping with embedded error control for density-driven flow in porous media, Water Resour. Res., 46, W08523, doi:10.1029/2009WR008229.

\section{Introduction}

[2] Modeling density-driven flow problems requires a coupled flow-transport numerical model where the equation of fluid conservation, Darcy's law and the advectiondispersion mass (or heat) transport equations are coupled by state equations linking density and viscosity to mass fraction (or temperature). Due to these nonlinearities, the simulations can require an excessive computational time and/or heavy equipments.

[3] The linearization of the coupled problem can be performed with Picard [Molson and Frind, 1994; Kolditz et al., 1998; Ackerer et al., 1999, 2004; Ackerer and Younes, 2008; Oltean and Buès, 2001; Mazzia and Putti, 2002, 2005; Woods and Carey, 2007] or Newton [Oldenburg and Pruess, 1995; Frolkovic and De Schepper, 2000; Johannsen et al., 2002] methods at each time step. In practice, the Picard scheme where flow and transport equations are solved sequentially is prevalent due to its simplicity. The Picard linearization allows the use of numerical methods and/or temporal discretizations that are specifically suited for achieving high accuracy for each kind of equations. In this work, the implicit time discretization is used for the flow equation whereas the explicit time discretization is preferred for the advective part of the transport equation. Indeed, high order explicit methods allow obtaining accurate and monotone solution with a minimum amount of numerical diffusion even in the presence of sharp fronts [Ackerer et al., 1999;

\footnotetext{
${ }^{1}$ Laboratoire d'Hydrologie et de Géochimie de Strasbourg, Université de Strasbourg, EOST, CNRS, Strasbourg, France.
}

Copyright 2010 by the American Geophysical Union. 0043-1397/10/2009WR008229
Ackerer and Younes, 2008; Mazzia and Putti, 2002, 2005]. Due to the stability constraint of the explicit scheme, different time step sizes can be used for flow and transport temporal discretizations.

[4] Adaptive time stepping is often used to reduce the computational cost of the simulation. Time stepping schemes with embedded error control were developed in many nonlinear problems like density-driven flow [Diersch, 1988; Diersch and Kolditz, 1998], incompressible Navier-Stokes problems [Turek, 1996], unsaturated flow [Tocci et al., 1997; Williams and Miller, 1999; Kavetski et al., 2001, 2002], elastoplastic consolidation in geomechanics [Sloan and Abbo, 1999], and reactive transport problems [Saaltink et al., 2004]. In this paper, different time stepping schemes are used to study the effect of temporal truncation errors for density-dependent flow problems.

[5] The Elder problem [Elder, 1966, Voss and Souza, 1987] is one of the most popular test used to benchmark density-driven codes and is chosen here to illustrate the effects of the temporal discretization. A review of prior work shows that there is a wide variation in published results. Various previous studies have shown the significant influence of the spatial discretization on the behavior of the solution [Oldenburg and Pruess, 1995; Boufadel et al., 1999; Oltean and Buès, 2001; Frolkovic and De Schepper, 2000]. Generally, the time discretization is based on (1) a constant time step [Frolkovic and De Schepper, 2000; Woods and Carey, 2007] which can lead to prohibitive CPU time, (2) an empirical time stepping which adapts the time step size according to the number of iterations required to achieve convergence [Mazzia and Putti, 2002; Oldenburg and Pruess, 1995; Putti and Paniconi, 1995], and (3) a time stepping based on local truncation error control [Diersch, 1988; Diersch and Kolditz, 1998]. 
[6] In this work, the Elder test problem is used to study the effect of time stepping for density-driven flow problems. To this aim, we compare the performance of (1) the standard iterative scheme with the empirical time stepping, (2) the iterative scheme using time stepping with embedded error control and (3) a non iterative scheme with time stepping based on truncation error control.

\section{Mathematical Models}

[7] The most common mathematical models of coupled fluid flow and mass (or heat) transport in porous media are based on the work of Bear [1972]. The proposed models have been widely discussed by Hassanizadeh and Leijnse [1988] and Diersch and Kolditz [1998, 2002] among others.

[8] The mass conservation of the fluid (water and dissolved salt) can be written in terms of equivalent fresh water head [Huyakorn et al., 1987],

$$
\begin{gathered}
\rho S_{s} \frac{\partial h}{\partial t}+\varepsilon \frac{\partial \rho}{\partial C} \frac{\partial C}{\partial t}+\rho \nabla \cdot\left[-\frac{\rho_{0} g}{\mu} \boldsymbol{k}\left(\nabla h+\frac{\rho-\rho_{0}}{\rho_{0}} \nabla z\right)\right]=0 \\
\boldsymbol{q}=-\frac{\rho_{0} g}{\mu} \boldsymbol{k}\left(\nabla h+\frac{\rho-\rho_{0}}{\rho_{0}} \nabla z\right)
\end{gathered}
$$

with $\rho$ the fluid density $\left[\mathrm{ML}^{-3}\right], S_{s}$ the specific mass storativity related to head changes $\left[\mathrm{L}^{-1}\right], h$ the equivalent freshwater head [L], $t$ the time [T], $\varepsilon$ the porosity $[-], C$ the solute mass fraction [M. salt/M. fluid], $\boldsymbol{q}$ the Darcy's velocity $\left[\mathrm{LT}^{-1}\right], \rho_{0}$ the density of the displaced fluid $\left[\mathrm{ML}^{-3}\right]$, $g$ the gravity acceleration [LT- $\left.{ }^{2}\right], \mu$ the fluid dynamic viscosity $\left[\mathrm{ML}^{-1} \mathrm{~T}^{-1}\right], \boldsymbol{k}$ the permeability tensor $\left[\mathrm{L}^{2}\right]$ and $z$ the depth [L].

[9] The mass transport of the dissolved salt in saturated porous media is described by the following convectiondispersion equation:

$$
\varepsilon \frac{\partial C}{\partial t}+\nabla \cdot(\boldsymbol{q} C)=\nabla \cdot(\boldsymbol{D} \cdot \nabla C)
$$

where $\mathrm{C}$ is the dimensionless mass fraction and the dispersion tensor $\boldsymbol{D}$ is given by

$$
\boldsymbol{D}=\varepsilon D_{m} \boldsymbol{I}+\left(\alpha_{L}-\alpha_{T}\right) \boldsymbol{q}_{i} \boldsymbol{q}_{j} /|\boldsymbol{q}|+\alpha_{T}|\boldsymbol{q}| \boldsymbol{I}
$$

with $\alpha_{L}$ and $\alpha_{T}$ the longitudinal and transverse dispersivities [L], $D_{m}$ the pore water diffusion coefficient $\left[\mathrm{L}^{2} \mathrm{~T}^{-1}\right], \varepsilon$ the porosity and $\boldsymbol{I}$ the unit tensor. The associated boundary conditions of the flow-transport system (1)-(3) are of Dirichlet, Neuman or mixed type.

[10] Flow and transport equations are coupled by state equations linking density and viscosity to mass fraction like

$$
\rho=\rho_{0}+\left(\rho_{1}-\rho_{0}\right) C, \text { and } \mu=\mu_{0}\left(\frac{\mu_{1}}{\mu_{0}}\right)^{C}
$$

with $\rho_{1}$ and $\mu_{1}$, respectively, density and viscosity of the injected fluid and $\mu_{0}$ the viscosity of the displaced fluid. Different state equations may be used for density or viscosity [Diersch and Kolditz, 1998].

\section{Time Stepping}

[11] The effect of time stepping for density-driven flow problems is studied using a code based on numerical methods that are specifically suited to achieve high accuracy for each kind of equation. As in [Ackerer and Younes, 2008], the Mixed Finite Element (MFE) method is used for the flow equation since it is locally conservative and produces accurate and consistent velocity field. The combination of the MFE method for flow and dispersion with high resolution methods for advection has been shown to be an effective tool for solving density dependent flow problems [Mazzia et al., 2001; Mazzia and Putti, 2002].

[12] For the transport equation, the explicit Discontinuous Galerkin (DG) method is used to discretize advection and combined (without time splitting procedure) with the Multipoint Flux Approximation (MPFA) method for the discretization of the dispersion equation [Younes and Ackerer, 2008]. The explicit DG method leads to a robust and accurate numerical scheme without suffering from numerical oscillations and with a minimum amount of numerical diffusion. The MPFA method is locally conservative and handles general irregular grids and heterogeneous domains [Aavatsmark, 2002].

\subsection{Scheme 1: The Iterative Scheme With Empirical Time Stepping}

[13] With this scheme, we iterate between flow and transport equations to progress from $t^{n}$ to $t^{n+1}$ as in the following steps [Ackerer et al., 2004].

[14] Step 1: The transport equation is solved first for $C_{k+1}^{n+1}$, using $\boldsymbol{q}_{k}^{n+1}$ the velocity at the time step $(n+1)$ and the previous iteration $k$ :

$$
\varepsilon \frac{C_{k+1}^{n+1}-C^{n}}{\Delta t^{n}}+\nabla \cdot\left(\boldsymbol{q}_{k}^{n+1} C^{n}\right)=\nabla \cdot\left(\boldsymbol{D}_{k}^{n+1} \cdot \nabla C_{k+1}^{n+1}\right)
$$

Note that advection is solved explicitly with the DG method to reduce numerical diffusion.

[15] Step 2: The fluid properties (density $\rho_{k+1}^{n+1}$ and viscosity $\left.\mu_{k+1}^{n+1}\right)$ are updated.

[16] Step 3: The flow equation is solved for $h_{k+1}^{n+1}$ using the recently obtained mass fractions $\mathrm{C}_{k+1}^{n+1}$ :

$$
\begin{aligned}
S \frac{h_{k+1}^{n+1}-h^{n}}{\Delta t^{n}}+\frac{\varepsilon}{\rho_{k+1}^{n+1}} \frac{\partial \rho}{\partial C} \frac{C_{k+1}^{n+1}-C^{n}}{\Delta t^{n}}+\nabla \\
\cdot\left[-\frac{\rho_{0} g}{\mu_{k+1}^{n+1}} \boldsymbol{k}\left(\nabla h_{k+1}^{n+1}+\frac{\rho_{k+1}^{n+1}-\rho_{0}}{\rho_{0}} \nabla z\right)\right]=0
\end{aligned}
$$

[17] Step 4: The velocity $\boldsymbol{q}_{k+1}^{n+1}$ is then calculated using $h_{k+1}^{n+1}$ and $C_{k+1}^{n+1}$ :

$$
\boldsymbol{q}_{k+1}^{n+1}=-\frac{\rho_{0} g}{\mu_{k+1}^{n+1}} \boldsymbol{k}\left(\nabla h_{k+1}^{n+1}+\frac{\rho_{k+1}^{n+1}-\rho_{0}}{\rho_{0}} \nabla z\right)
$$

This four-step sequence is repeated until convergence. For all performed test runs, the convergence behavior is dictated by the concentration errors. The relative maximum norm is used to constrain the largest absolute error on mass fractions across the entire domain:

$$
\frac{\max \left|\boldsymbol{C}_{k+1}^{n+1}-\boldsymbol{C}_{k}^{n+1}\right|}{\max \left(\boldsymbol{C}_{k+1}^{n+1}\right)} \leq \tau_{C}
$$

Different values for the tolerance $\tau_{C}$ are used in the literature, for example, $\tau_{C}=10^{-2}$ [Putti and Paniconi, 
1995], $\tau_{C}=10^{-4}$ [Woods and Carey, 2007] and $\tau_{C}=$ $10^{-6}$ [Mazzia and Putti, 2002].

[18] The time step control is of empirical type [see, e.g., Oldenburg and Pruess, 1995; Mazzia and Putti, 2002]. The time step size is adaptively adjusted according to the convergence behavior of the linearization scheme. At the end of each time step, the size of the following time step is increased by a factor $r_{1}>1.0$ if convergence was achieved in fewer than $n_{\text {inf }}$ iterations, and is decreased by a factor $r_{2}<1.0$ if convergence required more than $n_{\text {sup }}$ iterations. If convergence is not reached within a maximum number of iterations niter $_{\max }$, the current time step is repeated using a halved time step size. The values of $n_{\text {inf }}, n_{\text {sup }}, r_{1}, r_{2}$ and niter $_{\max }$ are chosen empirically and are fixed for the entire simulation. To avoid abrupt and dramatic changes in the time step size, the values $n_{\text {inf }}=4, n_{\text {sup }}=8, r_{1}=1.10, r_{2}=$ 0.80 and niter $_{\max }=20$ used by D'Haese et al. [2007] were found appropriate for all simulations performed in this work.

\subsection{Scheme 2: The Iterative Scheme With Time Stepping Based on Local Error Control}

[19] In this scheme, iterations between the transport and the flow equations are performed until the convergence of the nonlinear solver (i.e., criterion (9) is met). Then, the temporal truncation error is calculated to control the following time step size.

[20] Diersch [1988] and Diersch and Kolditz [1998] developed an adaptive predictor-corrector one-step Newton scheme where the size of the time step is automatically varied according to the local time truncation error. Sloan and Abbo [1999] and Kavetski et al. [2001, 2002] evaluated the local time truncation error by comparing two approximations of adjacent order of accuracy. The first-order Taylor series expansion gives

$$
\left.\boldsymbol{C}^{n+1}\right|_{(1)} \approx \boldsymbol{C}^{n}+\Delta t^{n+1} \dot{\boldsymbol{C}}^{n}
$$

where $\dot{\boldsymbol{C}}^{n}$ indicates time derivatives. The second-order approximation can be obtained by

$$
\begin{aligned}
\left.\boldsymbol{C}^{n+1}\right|_{(2)} & \approx \boldsymbol{C}^{n}+\Delta t^{n+1} \dot{\boldsymbol{C}}^{n}+\frac{\left(\Delta t^{n+1}\right)^{2}}{2} \ddot{\boldsymbol{C}}^{n} \\
& \approx \boldsymbol{C}^{n}+\frac{1}{2} \Delta t^{n+1}\left(\dot{\boldsymbol{C}}^{n}+\dot{\boldsymbol{C}}^{n+1}\right)
\end{aligned}
$$

where $\ddot{\boldsymbol{C}}^{n}$, the second order time derivatives is obtained by taking the derivative of equation (10). The difference between the previous approximations gives an estimation of the truncation error [Sloan and Abbo, 1999, Kavetski et al., 2002]:

$$
\boldsymbol{e}^{n+1} \approx\left|\boldsymbol{C}^{n+1}\right|_{(1)}-\left.\boldsymbol{C}^{n+1}\right|_{(2)}\left|=\frac{1}{2} \Delta t^{n+1}\right| \dot{\boldsymbol{C}}^{n}-\dot{\boldsymbol{C}}^{n+1} \mid
$$

where $\dot{\boldsymbol{C}}^{n+1}$ and $\dot{\boldsymbol{C}}^{n}$ are evaluated using the concentrations at previous times, yielding

$$
\boldsymbol{e}^{n+1} \approx \frac{1}{2}\left|\boldsymbol{C}^{n+1}-\left(\boldsymbol{C}^{n}+\frac{\Delta t^{n+1}}{\Delta t^{n}}\left(\boldsymbol{C}^{n}-\boldsymbol{C}^{n-1}\right)\right)\right|
$$

Note that the same truncation error can be obtained from the predictor-corrector scheme of Diersch [1988] and Diersch and Kolditz [1998] if we consider that the predicted con- centrations are $\boldsymbol{C}^{n}+\frac{\Delta t^{n+1}}{\Delta t^{n}}\left(\boldsymbol{C}^{n}-\boldsymbol{C}^{n-1}\right)$ and the corrected concentrations are the calculated concentrations $\boldsymbol{C}^{n+1}$.

[21] During the simulation the time step is accepted if the absolute error criterion is verified:

$$
\left|\boldsymbol{e}^{n+1}\right|<\gamma
$$

If this criterion is met, the next time step is calculated as in the work by Kavetski et al. [2001]:

$$
\Delta t^{n+1}=\Delta t^{n} \times \min \left(s \sqrt{\frac{\gamma}{\max \left|\boldsymbol{e}^{n+1}\right|}}, r_{\max }\right)
$$

where the time step size is directly controlled by the temporal truncation error tolerance $\gamma$. If the error criterion is not satisfied, the current time step is repeated using the latest error estimate

$$
\Delta t_{j+1}^{n+1}=\Delta t_{j}^{n+1} \times \max \left(s \sqrt{\frac{\gamma}{\max \left|\boldsymbol{e}^{n+1}\right|}}, r_{\min }\right)
$$

where $j$ indexes the recursive step size reduction, $r_{\max }$ and $r_{\min }$ are used to limit multiplication and reduction factors and often set equal to 2.0 and 0.1 respectively and $s=0.9$ a safety factor [Sloan and Abbo, 1999].

\subsection{Scheme 3: The Non Iterative Scheme With Time Stepping Based on Local Error Control}

[22] In this scheme, iterations between flow and transport equations are avoided by using the velocity at the old time level $\boldsymbol{q}^{n}$ in the transport equation (6). As previously, the time step size is adaptively adjusted according to the local time truncation error.

\section{Numerical Experiments}

[23] To compare efficiency and accuracy of the three schemes, we simulate the Elder problem on a PC with an Intel $2.6 \mathrm{GHz}$ processor. It is a free convection problem where flow is driven purely by fluid density differences in a $2 \mathrm{D}$ domain of $600 \mathrm{~m} \times 150 \mathrm{~m}$. The problem involves total density variations of $20 \%$ which makes it a strongly coupled flow case. The viscosity is assumed to be constant. The problem being symmetrical at $\mathrm{x}=300 \mathrm{~m}$, only one half of the domain is simulated. The parameters, initial and boundary conditions for the problem are given in Table 1 . The usual boundary conditions are used to compare our results with previous works, although the flow boundary conditions are not consistent with the transport boundary conditions [Hidalgo et al., 2009].

[24] The Elder test is simulated with the three described time stepping schemes to study the effect of temporal truncation errors on the numerical results. A triangular mesh of 16384 triangles $(128 \times 64 \times 2)$ is used for the simulation of the half domain. The orientation of the diagonals is optimized to avoid a non symmetric mesh and possible effects on the numerical results [Boufadel et al., 1999]. The discussion of the results is based not only on the mass fraction distribution, but also on the time step length, the total number of iterations, and the CPU time which are summarized in Table 2. The discussion of the existence of central upwelling or downwelling flow is still open for the Elder problem [Woods and Carey, 2007]. However, most of 
Table 1. Parameters and Boundary Conditions for the Elder Problem

\begin{tabular}{lc}
\hline \multicolumn{1}{c}{ Parameter or Condition } & Value \\
\hline Permeability & $\mathrm{k}_{\mathrm{x}}=\mathrm{k}_{\mathrm{z}}=4.84510^{-13} \mathrm{~m}^{2}$ \\
Porosity & $\varepsilon=0.1$ \\
Dispersivity & $\alpha_{L}=\alpha_{T}=0 \mathrm{~m}$ \\
Molecular diffusion coefficient & $D_{m}=3.56510^{-6} \mathrm{~m}^{2} / \mathrm{s}$ \\
Dynamic viscosity & $\mu=10^{-3} \mathrm{~kg} / \mathrm{m} / \mathrm{s}$ \\
State equation & $\rho=\rho_{0}+200 \mathrm{Cg} / \mathrm{m}^{3}$ \\
Initial conditions & $h(x, z)=0 \mathrm{~m},(x, z) \in[0,300 \mathrm{~m}] \times[0,150 \mathrm{~m}]$ \\
& $C(x, z)=0,(x, z) \in[0,300 \mathrm{~m}] \times[0,150 \mathrm{~m}]$ \\
Boundary conditions & $h(x, z)=0 \mathrm{~m}, x \in[0,1 \mathrm{~m}], z=150 \mathrm{~m}$ \\
& $\nabla h=0$, elsewhere \\
& $C(x, z)=1, x \in[150 \mathrm{~m}, 300 \mathrm{~m}], z=150 \mathrm{~m}$ \\
& $C(x, z)=0, x \in[0,300 \mathrm{~m}], z=0 \mathrm{~m}$ \\
& zero concentration gradient elsewhere \\
\hline
\end{tabular}

the published results show an central upwelling flow for this level of mesh refinement [Ackerer et al., 1999; Ackerer and Younes, 2008; Boufadel et al., 1999; Oltean and Buès, 2001; Kolditz et al., 1998; Oldenburg and Pruess, 1995] although central downwelling flow has been obtained for similar or even finer meshes [Diersch and Kolditz, 2002; Frolkovic and de Schepper, 2000].

[25] A reference simulation was run with the iterative scheme and a constant time step $\Delta t=0.1$ day. This time step is very small compared to simulations performed with constant time steps in the literature $(\Delta t=1 / 40$ year [Frolkovic and De Schepper, 2000; Woods and Carey, 2007] and $\Delta t=$ 1/12 year [Voss and Souza, 1987; Boufadel et al., 1999; Prasad and Simmons, 2005]). The stopping criterion is set to $\tau_{C}=10^{-3}$. Results at 20 years simulation time are plotted in Figure 1 and show a central upwelling. During the simulation, convergence is often reached within one iteration per time step. The whole simulation requires more than 73000 flowtransport solutions and spends more than 50000s of CPU time (Table 2). For the used spatial discretization, this solution is considered as the reference.

[26] A first set of simulations was run with the iterative scheme and empirical time stepping (scheme 1) using an initial time step of 0.1 day and the heuristic rules defined previously. Additional simulations were run with different values of the parameters involved in the heuristic rules without significant differences in the results. For example, the simulation with $r_{1}=1.30$ instead of 1.10 gives the same results and the total CPU time is varied by less than $5 \%$.

[27] Three simulations were performed with the following stopping criteria $\tau_{C}=10^{-3}, \tau_{C}=10^{-5}$ and $\tau_{C}=10^{-7}$ and the corresponding results are given in Figure 2 and Table 2. A central downwelling flow is found with both stopping cri-

Table 2. Summary of Numerical Indicators for the Simulations

\begin{tabular}{lccccc}
\hline & & & $\Delta \mathrm{t}_{\max }$ & Number of \\
& $\tau_{C}(-)$ & $\gamma(-)$ & $(\mathrm{d})$ & $\begin{array}{c}\text { CPU Time } \\
\text { Iterations }\end{array}$ & $(\mathrm{s})$ \\
\hline Constant $\Delta \mathrm{t}$ & $10^{-3}$ & - & 0.1 & 73352 & 52000 \\
Scheme 1 & $10^{-3}$ & - & 261 & 800 & 1680 \\
& $10^{-5}$ & - & 53 & 4000 & 3942 \\
\multirow{2}{*}{ Scheme 2 } & $10^{-7}$ & - & 15 & 13700 & 10901 \\
\multirow{2}{*}{ Scheme 3 } & $10^{-3}$ & $10^{-3}$ & 171 & 1709 & 1966 \\
& $10^{-3}$ & $10^{-4}$ & 51 & 4792 & 3882 \\
\end{tabular}

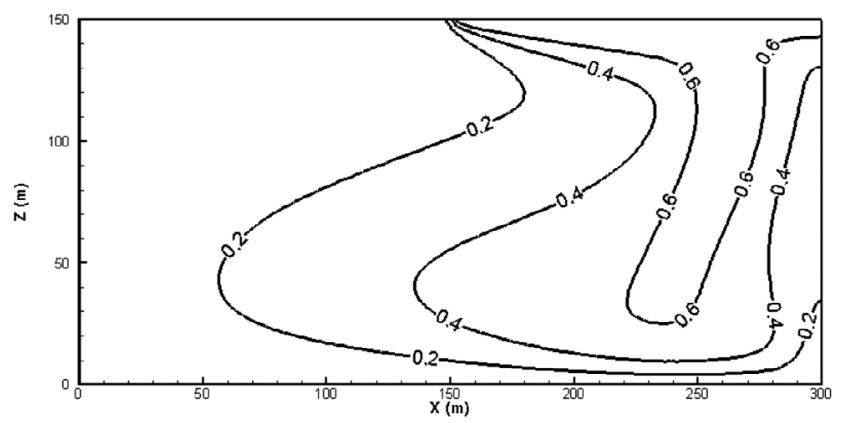

Figure 1. Concentration contours (at $t=20$ years) with the iterative scheme and a constant time step of 0.1 day $\left(\tau_{C}=10^{-3}\right)$.

teria $\tau_{C}=10^{-3}$ and $\tau_{C}=10^{-5}$. Small differences can be observed between the contour plots (see contour 0.2 in Figure 2). The time step size increases from the initial value 0.1 day to reach 261 days and 53 days respectively at the end of the simulation. During the period between 500 days and 1500 days of simulation time, the time step length is about 20 days for $\tau_{C}=10^{-3}$ and 7 days for $\tau_{C}=10^{-5}$. The whole simulation with $\tau_{C}=10^{-5}$ requires 2.5 times more CPU time than with $\tau_{C}=10^{-3}$ (Table 2). Figure 2 shows that the truncation error defined by equation (13) can be quite high (maximum value around 0.05 ) for $\tau_{C}=10^{-3}$. This truncation error remains important (maximum value around 0.005 ) with the small convergence criterion $\tau_{C}=10^{-5}$.

[28] Contrarily to the previous simulations, the results with the smallest stopping criterion $\tau_{C}=10^{-7}$ show a central upwelling flow at 20 years simulation time (Figure 2) which is consistent with the reference solution. The time step size is around 1.8 days for the period 500-1500 days, it reaches a maximum value of 15 days at the end of the simulation. In this case, the convergence criterion is very small, thus small time steps are required and therefore the temporal truncation error remains below $10^{-3}$ (Figure 2). The total CPU time for the simulation is more than 6 times more important than with $\tau_{C}=10^{-3}$ (Table 2).

[29] The second set of simulations is performed with scheme 2. The convergence criterion is set to $\tau_{C}=10^{-3}$ and the time step size selection is based on the temporal truncation error which was fixed to $\gamma=10^{-3}$ [Diersch and Kolditz, 1998] and $\gamma=10^{-4}$. The results show a central upwelling at 20 years simulation time (Figure 3) and no significant differences can be observed between the two mass fraction distributions. The time step size increases to reach a maximum value of 171 days for $\gamma=10^{-3}$ and 51 days for $\gamma=10^{-4}$. The whole simulation with $\gamma=10^{-4}$ requires the double of the CPU time used with $\gamma=10^{-3}$ (Table 2).

[30] Recall that the results of scheme 1 with both convergence criteria $\tau_{C}=10^{-3}$ and $\tau_{C}=10^{-5}$ show a central downwelling flow. Figure 3 shows that when the temporal truncation error is fixed to $\gamma=10^{-3}$ in addition to the convergence criterion of $\tau_{C}=10^{-3}$, the solution shows a central upwelling, consistent with the reference solution. Moreover, the total CPU time as well as the maximum time step length with scheme 1 and $\tau_{C}=10^{-5}$ are very close the values obtained with scheme 2 using $\tau_{C}=10^{-3}$ and $\gamma=10^{-4}$ 

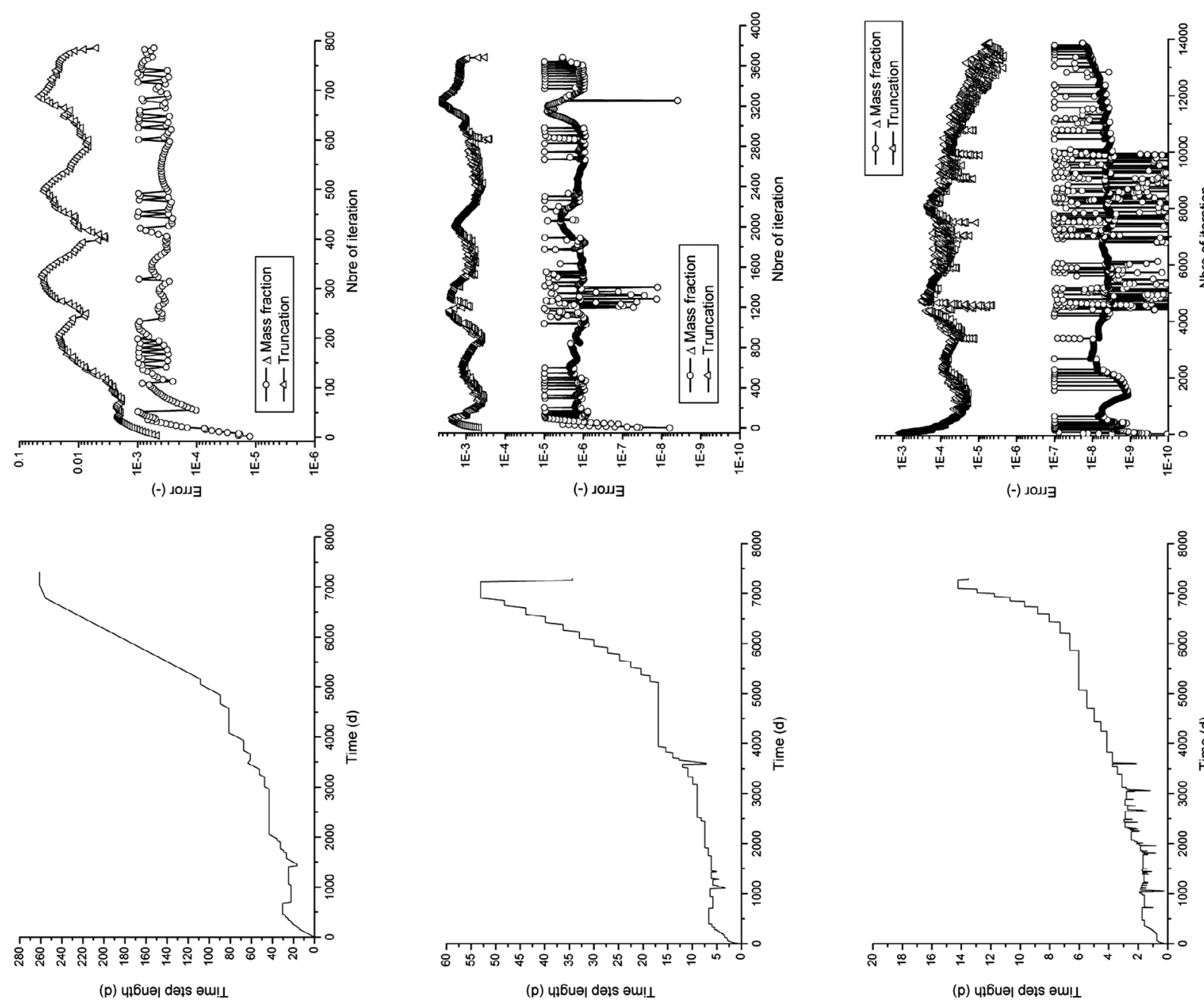

10

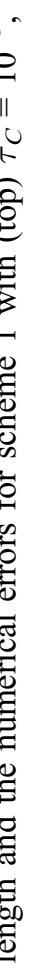
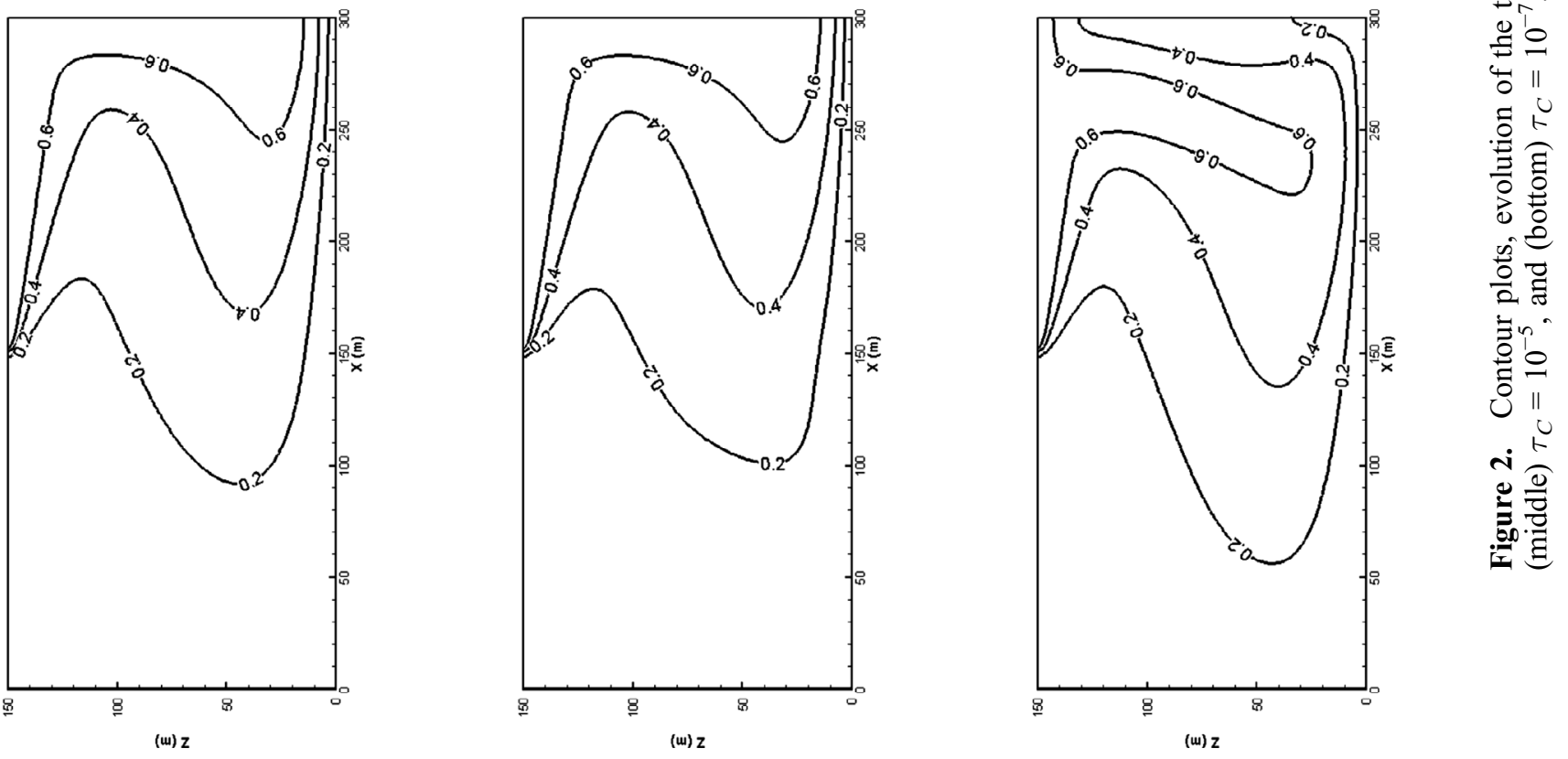

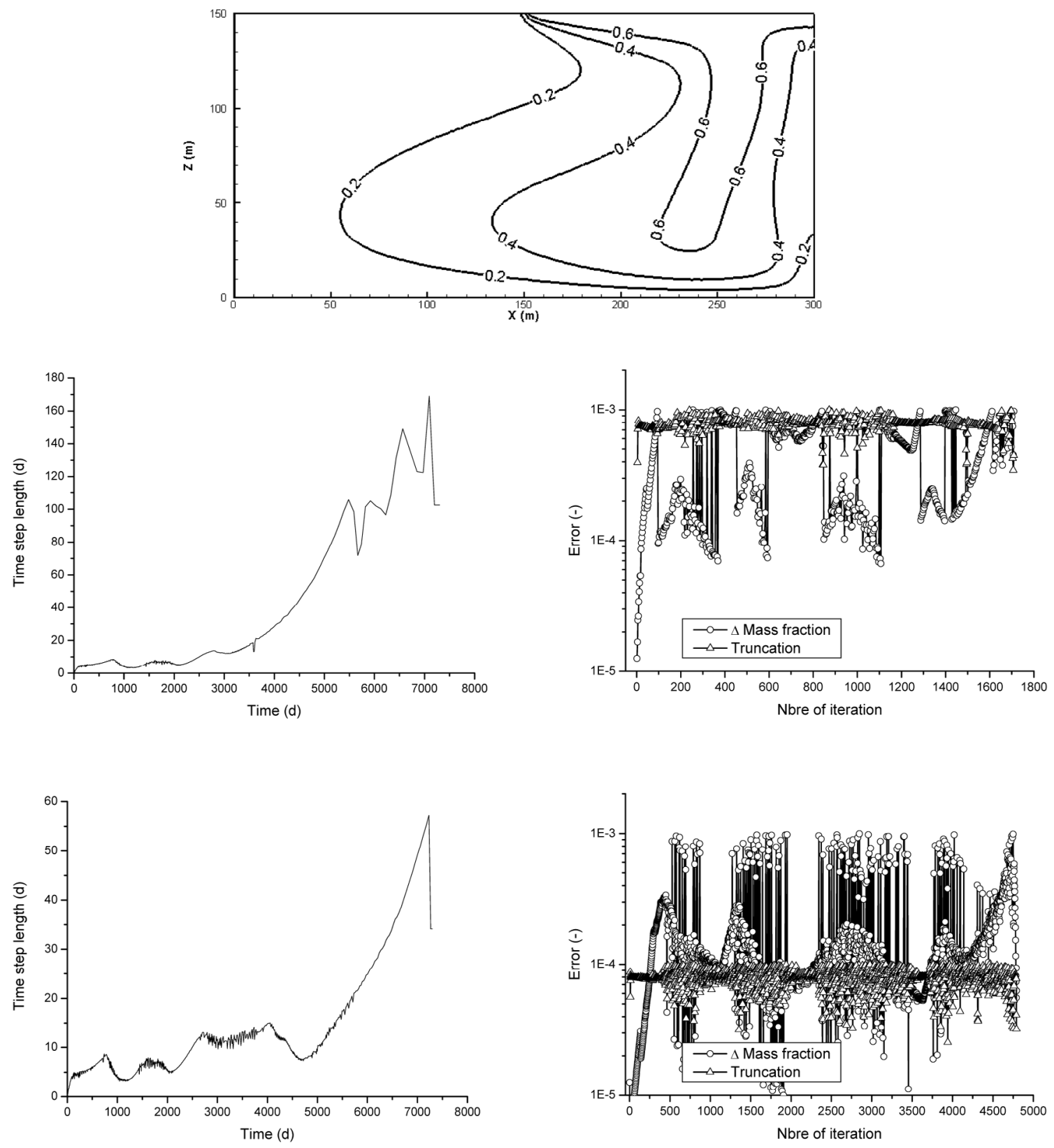

Figure 3. (top) Contour plots and evolution of the time step length and the numerical errors for scheme 2 for (middle) $\gamma_{C}=10^{-3}$ and (bottom) $\gamma_{C}=10^{-4}$.

(Table 2) but the results are completely different. Therefore, the results for these simulations are more sensitive to truncation errors than to convergence errors.

[31] Finally, the simulation is performed with scheme 3, i.e., without any control on the convergence error. In this case, the velocity at the old time level is used for the transport equation. Therefore, iterations between the flow and the transport equations are not necessary. The time step size selection is based on the temporal truncation error with a tolerance $\gamma=10^{-3}$. As before, the results show a central upwelling at 20 years simulation time and the time step size increases to reach a maximum value of 160 days (Figure 4).

[32] This scheme is the most efficient (Table 2) and the results are consistent with the reference solution. The number of flow-transport solutions is reduced because iterations are avoided and the time step is automatically varied according to the local time truncation error.

\section{Conclusion}

[33] Modeling flow and mass (or heat) transport in porous media may require a lot of CPU time due to the strongly coupled partial differential equations. Time stepping schemes are used to optimize the time step size and therefore, to reduce the computational cost. The empirical procedure based on the number of iterations required to achieve convergence is generally used. This procedure was compared to a procedure where the time step size selection is based on the temporal truncation error control. 

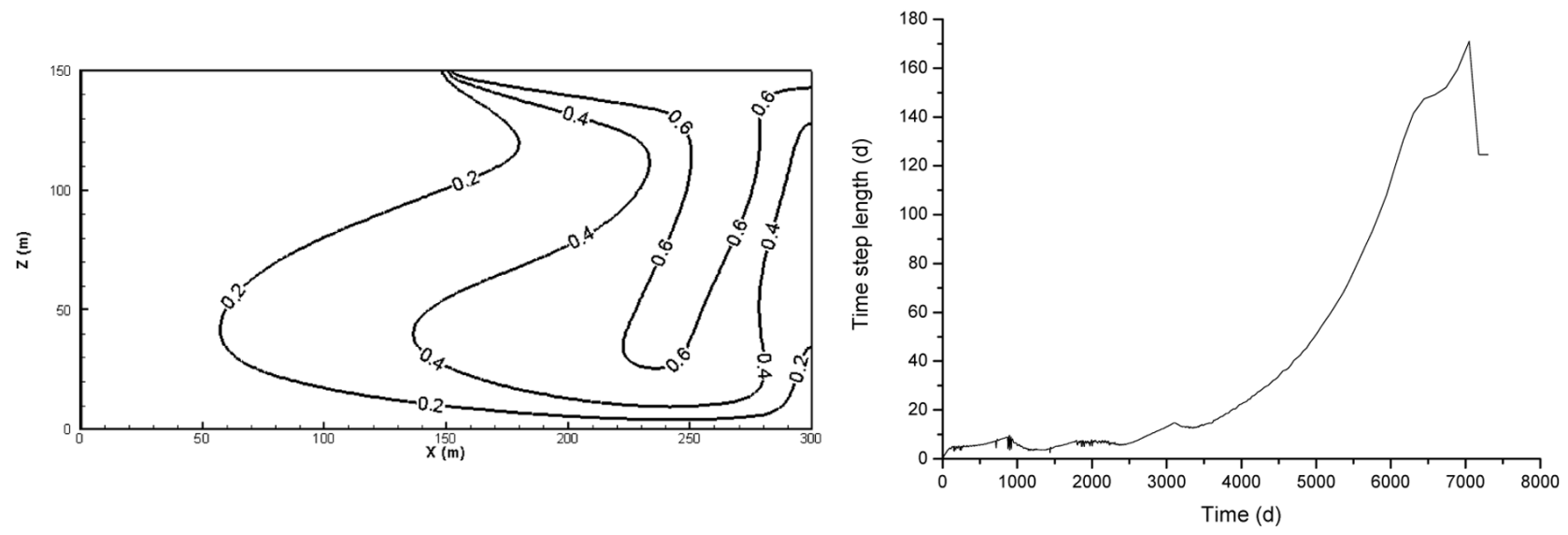

Figure 4. Contour plots and evolution of the time step length for scheme 3.

[34] Numerical experiments show the following.

[35] 1 . With the empirical scheme, the temporal truncation error may remain important which can lead to inaccurate results.

[36] 2. A non iterative scheme with proper time management can be faster and can lead to more accurate solution than the standard iterative procedure with the empirical time stepping.

[37] 3. The Elder test case is very sensitive. Time step discretization and management can have a significant effect on the results. The temporal truncation error can be considered as one of the reasons for the differences observed in Elder numerical results.

[38] The provided simulations show that error-controlled time stepping strategies are useful and should be considered as a leading option for solving density-driven flow problems.

\section{References}

Aavatsmark, I. (2002), An introduction to multipoint flux approximations for quadrilateral grids, Comput. Geosci., 6, 405-432, doi:10.1023/ A:1021291114475.

Ackerer, P., and A. Younes (2008), Efficient approximations for the simulation of density driven flow in porous media, Adv. Water Resour., 31, 15-27, doi:10.1016/j.advwatres.2007.06.001.

Ackerer, P., A. Younes, and R. Mosé (1999), Modeling variable density flow and solute transport in porous medium: 1. Numerical model and verification, Transp. Porous Media, 35(3), 345-373, doi:10.1023/ A:1006564309167.

Ackerer, P., A. Younes, and M. Mancip (2004), A new coupling algorithm for density-driven flow in porous media, Geophys. Res. Lett., L12506, doi:10.1029/2004GL019496.

Bear, J. (1972), Dynamics of Fluids in Porous Media, Elsevier, New York.

Boufadel, M. C., M. T. Suidan, and A. D. Venosa (1999), Numerical modelling of water flow below dry salt lakes: Effect of capillarity and viscosity, J. Hydrol., 221, 55-74, doi:10.1016/S0022-1694(99)00077-3.

D’Haese, C. M. F., M. Putti, C. Paniconi, and N. E. C. Verhoest (2007), Assessment of adaptive and heuristic time stepping for variably saturated flow, Int. Numer. Methods Fluids, 53, 1173-1193, doi:10.1002/ fld.1369.

Diersch, H.-J. (1988), Finite element modelling of recirculation densitydriven saltwater intrusion processes in groundwater, Adv. Water Resour., 11, 25-43, doi:10.1016/0309-1708(88)90019-X.

Diersch, H. J., and O. Kolditz (1998), Coupled groundwater flow and transport: 2. Thermohaline and 3D convection systems, Adv. Water Resour., 21, 401-425, doi:10.1016/S0309-1708(97)00003-1.

Diersch, H. J., and O. Kolditz (2002), Variable-density flow and transport in porous media: Approaches and challenges, Adv. Water Resour., 25, 899-944, doi:10.1016/S0309-1708(02)00063-5.
Elder, J. W. (1966), Numerical experiments with a free convection in a vertical slot, J. Fluid Mech., 24, 823-843, doi:10.1017/S0022112066001022.

Frolkovic, P., and H. De Schepper (2000), Numerical modelling of convection dominated transport coupled with density driven flow in porous media, Adv. Water Resour., 24, 63-72, doi:10.1016/S0309-1708(00) 00025-7.

Hassanizadeh, S., and A. Leijnse (1988), On the modeling of brine transport in porous media, Water Resour. Res., 24(3), 321-330, doi:10.1029/ WR024i003p00321.

Hidalgo, J. J., J. Carrera, and A. Medina (2009), Role of salt sources in density-dependent flow, Water Resour. Res., 45, W05503, doi:10.1029/ 2008WR007679.

Huyakorn, P., P. Anderson, J. Mercer, and H. White (1987), Saltwater intrusion in aquifers: Development and testing of a three-dimensional finite element model, Water Resour. Res., 23, 293-312, doi:10.1029/ WR023i002p00293.

Johannsen, K, W. Kinzelbach, S. Oswald, and G. Wittum (2002), The saltpool benchmark problem-numerical simulation of salt water upconing in a porous medium, Adv. Water Resour., 25, 335-348, doi:10.1016/S03091708(01)00059-8.

Kavetski, D., P. Binning, and S. W. Sloan (2001), Adaptive time stepping and error control in a mass conservative numerical solution of the mixed form of Richards equation, Adv. Water Resour., 24(6), 595-605, doi:10.1016/S0309-1708(00)00076-2.

Kavetski, D., P. Binning, and S. W. Sloan (2002), Adaptive backward Euler time stepping with truncation error control for numerical modelling of unsaturated fluid flow, Int. J. Numer. Methods Eng., 53, 1301-1322, doi:10.1002/nme.329.

Kolditz, O., R. Ratke, H. J. Diersch, and W. Zielke (1998), Coupled groundwater flow and transport: 1 . Verification of variable-density flow and transport models, Adv. Water Resour., 21, 27-46, doi:10.1016/ S0309-1708(96)00034-6.

Mazzia, A., and M. Putti (2002), Mixed finite element and finite volume discretization for heavy brine simulation in groundwater, J. Comput. Appl. Math., 147, 191-213, doi:10.1016/S0377-0427(02)00433-8.

Mazzia, A., and M. Putti (2005), High order Godunov mixed methods on tetrahedral meshes for density driven flow simulations in porous media, J. Comput. Phys., 208, 154-174, doi:10.1016/j.jcp.2005.01.029.

Mazzia, A., L. Bergamaschi, and M. Putti (2001) On the reliability of numerical solutions of brine transport in groundwater: Analysis of infiltration from a salt lake, Transp. Porous Media, 43, 65-86.

Molson, J. W., and E. O. Frind (1994), SALTFLOW: Density Dependent Flow and Mass Transport Model in Three Dimensions, User Guide, Waterloo Cent. for Groundwater Res., Univ. of Waterloo, Ontario.

Oldenburg, C., and K. Pruess (1995), Dispersive transport dynamics in a strongly coupled groundwater-brine flow system, Water Resour. Res., 31, 289-302, doi:10.1029/94WR02272.

Oltean, C., and M. A. Buès (2001), Coupled groundwater flow and transport in porous media. A conservative or non-conservative form?, Transp. Porous Media, 44(2), 219-246, doi:10.1023/A:1010778224076.

Prasad, A., and C. T. Simmons (2005), Using quantitative indicators to evaluate results from variable-density groundwater flow models, Hydrogeol. J., 13(5-6), 905-914, doi:10.1007/s10040-004-0338-0. 
Putti, M., and C. Paniconi (1995), Picard and Newton linearization for the coupled model for saltwater intrusion in aquifers, Adv. Water Resour., 18(3), 159-170, doi:10.1016/0309-1708(95)00006-5

Saaltink, M. W., J. Carrera, and S. Olivella (2004), Mass balance errors when solving the convective form of the transport equation in transien flow problems, Water Resour. Res., 40, W05107, doi:10.1029/ 2003WR002866.

Sloan, S. W., and A. J. Abbo (1999), Biot consolidation analysis with automatic time stepping and error control. Part 1: Theory and implementation, Int. J. Numer. Anal. Methods Geomech., 23, 467-492, doi:10.1002/(SICI) 1096-9853(199905)23:6<467::AID-NAG949>3.0.CO;2-R.

Tocci, M. D., C. T. Kelley, and C. T. Miller (1997), Accurate and economical solution of the pressure-head form of Richards' equation by the method of lines, Adv. Water Resour., 20(1), 1-14, doi:10.1016/S0309 1708(96)00008-5

Turek, S. (1996), A comparative study of some time-stepping techniques for the incompressible Navier-Stokes equations: From fully implicit nonlinear schemes to semi-implicit projection methods, Int. J. Numer Methods Fluids, 22(10), 987-1011, doi:10.1002/(SICI)1097-0363 (19960530)22:10<987::AID-FLD394>3.0.CO;2-7.
Voss, C. I., and W. R. Souza (1987), Variable density flow and solute transport simulation of regional aquifers containing a narrow freshwatersaltwater transition zone, Water Resour. Res., 23, 1851-1866, doi:10.1029/WR023i010p01851.

Williams, G. A., and C. T. Miller (1999), An evaluation of temporally adaptive transformation approaches for solving Richards' equation, Adv. Water Resour., 22(8), 831-840, doi:10.1016/S0309-1708(98) 00048-7

Woods, J. A., and G. F. Carey (2007), Upwelling and downwelling behavior in the Elder-Voss-Souza benchmark, Water Resour. Res., 43, W12403, doi:10.1029/2006WR004918.

Younes, A., and P. Ackerer (2008), Solving the advection-dispersion equation with discontinuous Galerkin and multipoint flux approximation methods on unstructured meshes, Int. J. Numer. Methods Fluids, 58(6), 687-708, doi:10.1002/fld.1783.

P. Ackerer and A. Younes, Laboratoire d'Hydrologie et de Géochimie de Strasbourg, Université de Strasbourg, EOST, CNRS, 1 rue de Blessig, F67000 Strasbourg, France. (ackerer@unistra.fr) 